الفلبين وأوضاع المسلمين بها فى ظل الاحتلال الأمريكى

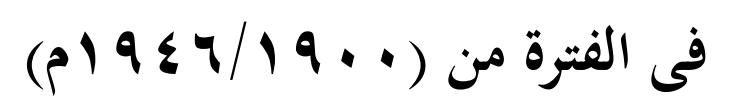

$$
\text { للباحث محمد على سعد }
$$




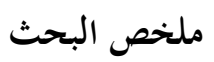

الحمد لله الذى جعل العلماء مصابيح كالنجوم في السماء ،يهتدى بهم فن ظلمات

الجهل، فيدلون العباد على ماينفعهم في دينهم ودنياهم والصلاة والسلام على النبى الكريم وبعد،6،

فإن موضوع هذا البحث يتمحور حول الفلبين وأوضاع المسلمين بها فن ظل الاحتلال

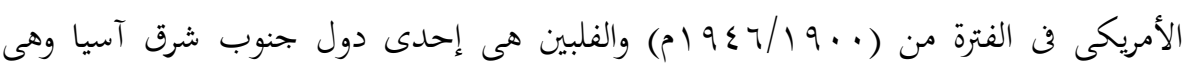
تضم أقلية مسلمة في جنوبها ،والتى دائما ما تتعرض تلك الأقلية إلى القهر والاعتداء والظلم ، ومن هذا المنطلق الديني، كان هذا البحث الذى يُلقى الضوء على ماعاناه المسلمون في الفلبين

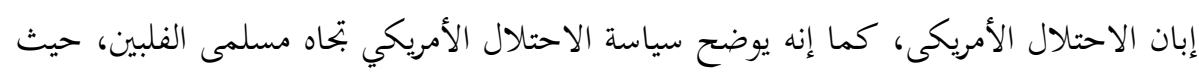
ظهرت في فترة الاحتلال الأمريكي تجاه مسلمي الفلبين بعض الآثار الضارة للسياسات الاستعمارية والإمبريالية علي الإسلام والمسلمين في الفلبين منها التنصير الأمريكى للمسلمين في الفلبين، وفقدان التماسك والشعور الإسلامى، وفقدان السيادة الإسلامية على معظم الإمارات والسلطنات، والاستيلاء على الأراضى الزراعية،وإهمال التعليم وغيرها من الآثار الضارة للسياسات الاستعمارية الأمريكية.

\section{ملخص الإنجليزى}

Praise be to Allah, The one who liken,s scholars to lanterns lighting as stars in in the sky, and by whom people in the darknesses of sheer ignorance ,they also lead servants of Allah to all what benefit them pertaining their religion and worldly things .thus,all prayers and peace be upon our prophet Muhammad. The subject of this research revolve around 
Philippines and muslims circumstances under the occupation of the USA(1900/1946) Philippine sisane of the southeast countries which includes a minority of muslims in its south .By the same token, this minority is always subjected to constraint, assault and oppression proceeding from this religious point, there it is the research which sheds the light on sufferings of Muslims in the Philippines during the American occupation .In addition, it shows the occupation policy towards them .Hence, some of the harmful effects of the colonialistic

and imperialistic policies were apparent during this period over the Islam and muslims, of these hateful policies were the American act of Christianization, the loss of Islamic harmony and coordination, the lack of Islamic control over their territories, the Seizure of of agricultural lands, the negligence of education and many other bad effects of these imperialistic policies of America. 


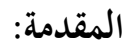

الحمدلله الذى منَّ علينا بنعمة الإسلام ،وشرفنا بكتابه العزيز الذى أنزل فيه (إن الدين عند الله الإسلام )،جعل من طلب العلم سبيلاً إلى رحته ،وطريقاً إلى جنته ،والصلاة والسلام على النور الخالد محمد (صلى الله عليه وسلم )خير الأنام ،الذى ورث من الجممال أهاه ،ومن الكمال أعلاه ،ومن الجلال أحسنه ،ومن التواضع أعذبه ،تدرَّج فن مراتب الأخلاق فوصفه الله في علاه

$$
\text { بقوله: (وإنك على خلق عظيم ). }
$$

فتعد الفلبين إحدى دول جنوب شرق آسيا ،وهى تحتوى على أقلية إسلامية يتمركز معظمها في جنوب الفلبين ،وهذه الأقلية تتعرض دائماً للقهر والاعتداء والظلم من قبل مواطنى الفلبين المسيحيين والذين يتركزون في شمال الفلبين ،ولم يكن هذا العداء وليد اللحظة وإنما كان نتاجاً لمخلفات الاستعمار السيئة ذلك الاستعمار الذى تعرضت له الفلبين عبر العصور المختلفة بداية من الاستعمار الأسبانى مروراً بالاحتلال الابحليزى وختاماً بالاحتلال الأمريكى،فقد كانت جزر الفلبين محتلة من قبل الاستعمار الاسبانى لمدة ثلاثة قرون تقريباً ،إلى آن قامت ثورة في

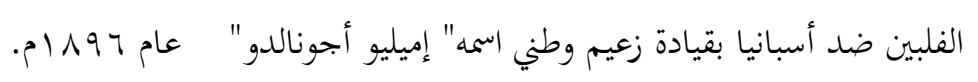

وكان دعاة الاستعمار والتوسع كثيرين في الولايات المتحدة الأمريكية خاصة في الكوبحرس الأمريكي وعند أصحاب رؤوس الأموال على حساب أسبانيا، وقد شنت الصحافة الأمريكية، وخحاصة الصحف التي كان يملكها إمبراطور الصحافة الأمريكي وليم هيرست" Hurst "، حملة تشهيرية عشواء على أسبانيا مدعية -وكعادتا لليوم- الدفاع عن حقوق الإنسان وعن الديمقراطية ومبرزة مساوئ الاستعمار الأسباني خاصة في كوبا، بعدما غرقت سفينة أمريكية في ميناء هافانا في كوبا الأسبانية وفى ظروف غامضة ومشبوهة مما جعل أمريكا تُعلن الحرب على أسبانيا في عام 11 191 م، ولم تنتظر حتي بتُري تحقيقاً فن أسباب الحادث، وكما هو معروف انتصرت الولايات المتحدة في الحرب. 
وبناءً على ذلك انتقلت السيادة على الفلبين من أسبانيا إلى الولايات المتحدة الأمريكية

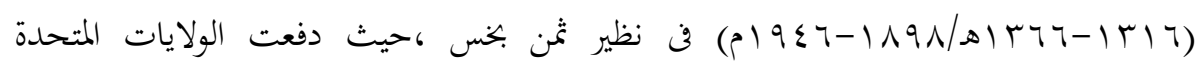

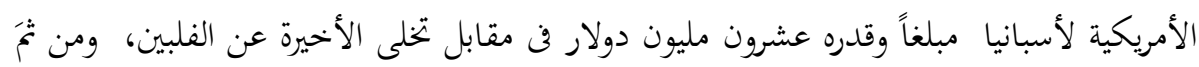
حلت أمريكا محل الأسبان في حكم هذه البلاد، واتبعت الولايات المتحدة نفس سياسة أسبانيا في ملاحقة المسلمين ومصادرة أراضيهم ،فأصدرت قانوناً يقضى بأن كل من لا يملك رخصة

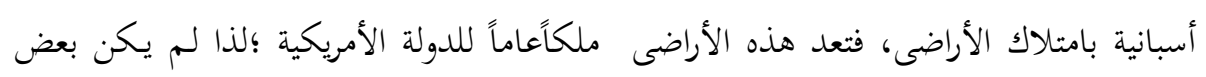

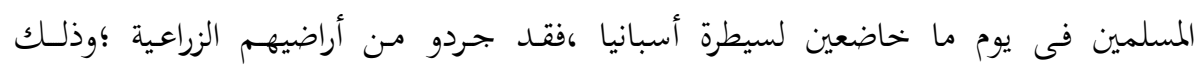
لكوفم دولة مستقلة لا ترتبط بالمتلين الأسبان.

كما أصـدرت الولايات المتحـدة قراراً بتخفيض ملكية الأرض الزراعية للمواطن الفلبينى المسلم، فلا يجوزله أن يمتلك أكثر من أربعة هكتارات، هذا لمن كان يملك حجة ملكية أسبانية، بينما منحت المهاجرين الجدد من مختلف أنحاء العالم المسيحى فرصة امتلاك الأرض الزراعية ،وتبعاً

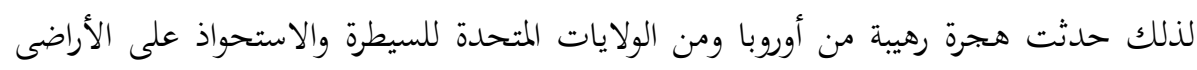
الزراعية التى صادرتما الدولة المحتلة وهى أمريكا.

بالإضافة إلى محاولة الأمريكيين تنصير المسلمين بالإكراه والقوة ، بينما استمرت المقاومة

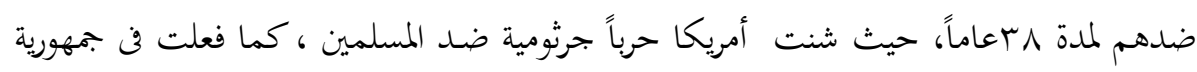
فيتنام بعد ذلك، فنشرت الجدرى والحصبة والكوليرا والطاعون في جزر مينداناو وسولو في سنة

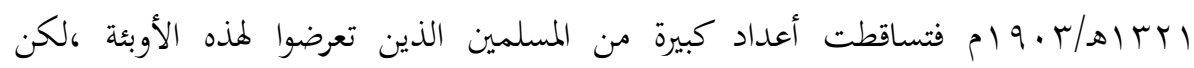
الأمريكان في آخر الأمر قد تساحوا مع المسلمين عكس الأسبان.

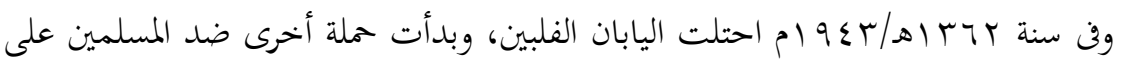
يد القوات اليابانية، ومع انتهاء الحرب العالمية الثانيةعام 9 المام اضطرت أمريكا إلى إعطاء الفلبين الاستقلال، بعد أن هيأت حكومة صليبية لا تقل وحشية عن الأسبان أو المولنديين

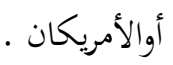


مشكلة البحث:

يُرجع البعض واقع المسلمين فن الفلبين إلى عوامل كثيرة منها :عوامل سياسية واقتصادية واجتماعية، ولقد أنتهجت الولايات المتحدة الأمريكية في الفلبين الوسائل التالية ضد الإسلام والمسلمين وهى:

1- التنصير الأمريكى للمسلمين فن الفلبين : فلقد حاول الأمريكيون تنصير المسلمين ومن أجل

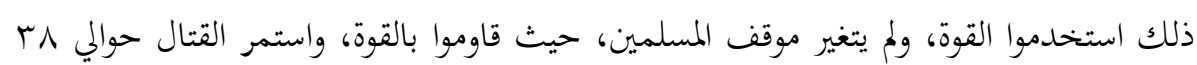
عاماً قتل فيها عدة آلاف من المحاهدين، وتمادي الغزاة فشنوا حرباً جرثومية، واجتاحت الأوبئة

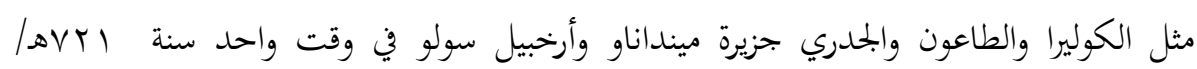
. PIrT

ب-فقدان المسلمين لحيازة الكثير من أراضيهم: فقد وضعت الولايات المتحدة الأمريكية قانوناً لنظام

حيازة الأراضى فى الجزر الفلبينية ،كان من نتيجته فقدان المسلمين لكثير من أراضيهم التى ورثوها عن أجدادهم، وسقطت فن أيدى المسيحيين الكاثوليك . r-ضياع التماسك والشعور الإسلامى: حيث قامت الولايات المتحدة الأمريكية عن طريق اتباع السياسة الاستعمارية التقليدية (فرق تسد) مهدف القضاء على التماسك الذى ظل طيلة القرون الماضية بمثابة السد المنيع ضد المحاولات الاستعمارية للفلبين.

ع -فقدان السيادة الإسلامية على معظم الإمارات والسلطنات: كانت كل الإمارات والسلطنات الإسلامية مستقلة ذات سيادة إسلامية، ولكن دوامة الحكم الأمريكي قد ابتلعتها جميعاً. ه-إهمال التعليم: كان التعليم آنذاك تحت إشراف الإرساليات التنصيرية المسيحية، وهذا ماجعل الثقافة الإسلامية تتقهقر والجهل يسود بين أبناء المسلمين. 


\section{أهداف البحث:}

يستهدف هذا البحث الوصول إلى الأهداف التالية:

رصد كفاح المسلمين ضد الأمريكان والمشاكل التى تعرض لها المسلمون، والتركيزعلى مظاهرالقوة والضعف وحملات الإبادة التى تعرض لها المسلمون من الاحتلال الأمريكى. r-إلقاء نظرة تاريخية على موقف المسلمين من الاحتلال الأمريكى.

أوضاع المسلمين فن الفلبين فن ظل الاحتلال الأمريكي فن الفترة من ( .99/1 / 9 (م) يعد مسلمو الفلبين من أكثر الجماعات في آسيا التي تتعرض لأشكال مختلفة من القمع الجماعي منذ القرن العاشر الهجرى/ السادس عشر الميلادي، فلقد ظلوا يقاتلون الأسبان علي مدي بrr عاماً، ثم الأمريكان أكثر من أربعين عاماً، واليابانيين لفترة خمس سنوات وكذلك سكريك البريطانيين والهولنديين علي فترات متقطعة، ونتيجة لذلك ذاقت الحركات الإسلامية في الفلبين

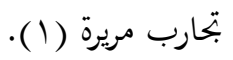

وقد جاءت سياسة الاستعمار الأمريكي الجديد بأسلوب مغاير تماماً، فنادرا ما لجأ الأمريكان إلى استعمال العنف ، وقد رفعوا شعاراً واضحاً "إننا لم نات لنهزم بل لنحرر" ولكن إلى لي لئل

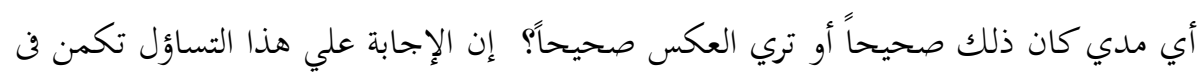
بيان اليخاندرو ميلكور (r)، الذي ألقاة أمام مؤتمر (الفلبين مشكلاتما والفرص المتاحة أمامها)

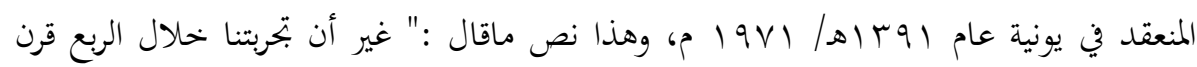
الأخير تشير إلى أنه بالرغم من أصدقائنا العديدين بما فيهم الشعب الأمريكي، فإن جهودنا لرفع مستوي معيشة الشعب الفلبيني ليست غير كافية فحسب بل إفا مخيبة للآمال أيضاً،وكشفت دراستنا لهذه الفترة الحرجة أننا نحن مصدر كثير من المشكلات التي نواجهها وإن كان بعضها يمكن بيكية رد أسبابه المباشرة إلى سنوات الاستعباد الاستعماري الحرجة، وأن مصدر كثير من المشكلات التي نواجهها يمكن رد أسبابه المباشرة إلى سنوات الاستعباد الاستعماري الطويلة التي ما تزال بصمات 
رواسبها وآثارها واضحة حتي يومنا هذا علي وجه ثقافة الفلبين ومؤسساتا وما هو أسوأ من التطور الاقتصادي الاستعماري الطابع فن بلادنا، يعود ذلك إلى الإدارة الاستعمارية القديمة قبل توحيد بمموعة جزر الفلبين تحت حكم واحد.

وبعد أن كانت مؤلفة من ممالك مستقلة وسلطنات قوية في جنوب الفلبين، فإن هذه

الإدارة الاستعمارية قد حرصت علي ألا تعطي الأهالي أي شكل من أشكال القضية غير ما

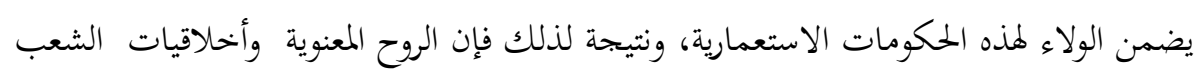
هبطت بصورة واضحة، وخبأ الحماس الذي كان في الثقافة الأصلية، ولم تكن القضية بالنسبة لمسلمي جنوب الفلبين بالذات، تتمثل في بجرد فقدان الشخصية بل إفم كانوا قد فقدوا سيادتم

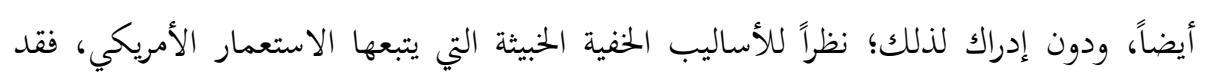
المسلمون تلك السيادة التي بنحوا في الحفاظ عليها عبر القرون الطويلة من الغزو والحصار الأسباني، وهكذا قبل أن يستيقظ الناس وجدوا أنفسهم داخل دوامة سيادة الحكم الأمريكي،وبدا

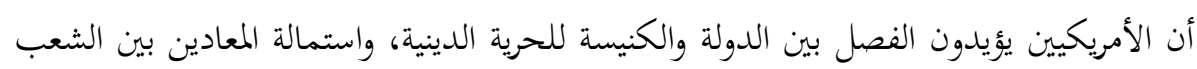
المهزوم، وإغراء غير المتدينين بالتعاون معهم، لكن الحقيقة أن الحكم الأمريكي أعطي كل التأييد والتشجيع لمختلف البعثات التنصيرية للإطاحة بالمسلمين الذين لم يكونوا علي أدني استعداد لمثل هذه المواجهة ،وقد كانت كافة السياسات التعليمية، والاجتماعية، والثقافية، الموجهة نخو المسلمين في الفلبين، تستهدف استئصال الروابط الإسلامية سياسياً، وثقافياً، تمهيداً للمناورة للهدف الحقيقي وهو إخراجةم من دين الإسلام"(").

حيث ترك الاحتلال الأمريكي آثاراً سلبية علي الفلبين بشكل عام وعلي المسلمين بشكل خاص في جنوب الفلبين، فعلي الرغم من قصر الفترة الزمنية التي ظل فيها الاحتلال الأمريكي

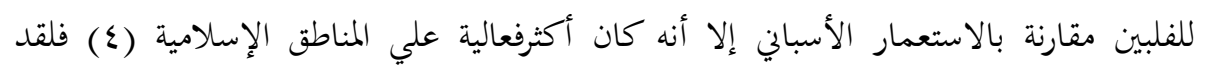
استفاد الأمريكيون من بحربة الأسبان القمعية ضد الجزر الجنوبية ،مما دفعهم إلى تغيير هذه السياسة والتحول إلى سياسة (المكر) حيث أقنعوا المسلمين بأفم جاءوا لعلاج أخطاء الأسبان 
وحدث على إثر ذلك اتفاقيات مثل اتفاقية (فرانك كاربنتر )الحاكم العام الأمريكى فن الفلبين(0).

وفن الحقيقة ظهرت في فترة الاحتلال الأمريكي للفلبين بعض الآثار الضارة للسياسات

الاستعمارية والإمبريالية علي الإسلام والمسلمين في الفلبين منها (†):

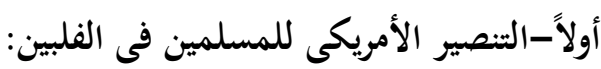

في الواقع عان المسلمون من سياسة الاحتلال الأمريكى حيث حاول الأمريكيون تنصير

المسلمين واستخدموا لذلك القوة، ولم يتغير موقف المسلمين، الذين قاوموا بالقوة، واستمر القتال حوالي مب عاماً قتل فيها عدة الأف من المحاهدين، وتمادي الغزاة فشنوا حرباً جرثومية، واجتاحت الأوبئة مثل الكوليرا والطاعون والحدري جزيرة مينداناو وأرخبيل سولو في وقت واحد سنة

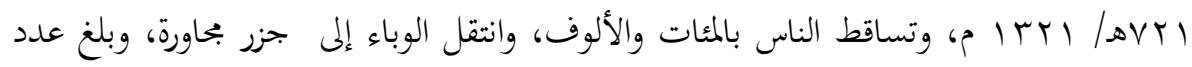
ضحايا الأوبئة أكثر من . T ألف علي حساب التقارير الغربية نفسها، وأهمها تقرير للحنة تافت(V)، وأدركت أمريكا عبث المحاولات فلانت وعقدت معاهدة مع المسلمين، احترمت خلالها دينهم وأسلوب حياهم وتكونت لهم دولة تحت الإدارة الأمريكية وبذلك استطاعت الولايات المتحدة دخول المناطق الإسلامية ،ولكن الحاكم العام الأمريكى (فرانك مورفن كاربنتر )جعل المسلمين تابعين لوزارة الداخلية الفلبينية عام عهب أهـ/ هـو ام في الفترة الانتقالية

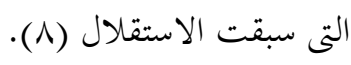

كما للجأت الولايات المتحدة إلى حيلة لإضعاف الوجود الإسلامي فن جنوب الفلبين من خلال خطة لتهجير المسيحيين إلى هناك، مما أدى إلى تحول سكانى نوعى لصالح المسيحيين لتصل نسبتهم إلى ـ\% بينما تراجعت نسبة المسلمين إلى •ـ\%فقط ،ليصل عددهم حالياً حوالى

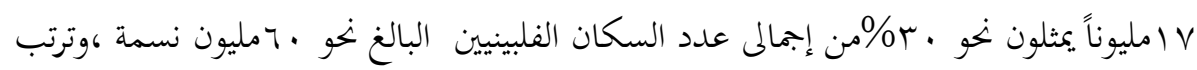
على ذلك فرص سياسية واقتصادية واجتماعية وتعليمية وثقافية لاتتناسب مع الواقع الإسلامى فن 
الجنوب ،فضلاً عن اتباع سياسة إسناد المناصب الحكومية العليا للمسيحيين الوافدين ، الأمر الذى أدى إلى سخط المسلمين خاصة مع استمرار اعتماد الحكومات الفلبينية المتعاقبة لهذه السياسة منذ استقلال البلاد عام 7 أو إمحتى الآن (9).

وقد ظل فرانك كاربنتر(الحاكم العام الأمريكى فن الفلبين ) متقلداً إدارة شؤون المسلمين

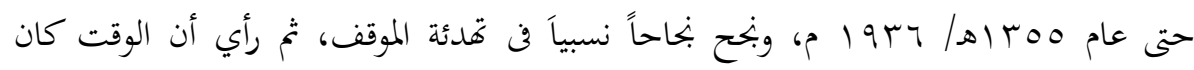
مناسباً لاستخدام سلاح آخر لكسب عاطفة وشعور المسلمين وهو السلاح الثقافي؛ ذلك أن أن اهن سلاح القوة التي لجأ إليه الأسبان، وكذلك الأمريكان فن المراحل الأولي لم تفدهم ولم يجلب لهم بحاحاً، أما السلاح الثقافي فهو يحارب العقل ويغير الأفكار والابحاهات، حتي عبرت الإدارة الأمريكية عن شكرها لكاربنتر علي جهده المتواصل وبحاحه في وضع الأسس القوية لتحويل المسلمين إلى طريق الحياة النصرانية(· (1).

وعلي الرغم من ذلك فقد تمكن فرانك كاربنتر من إرضاء بعض الزعماء المسلمين الذين

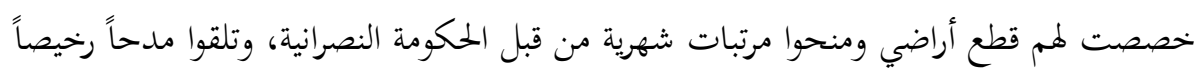

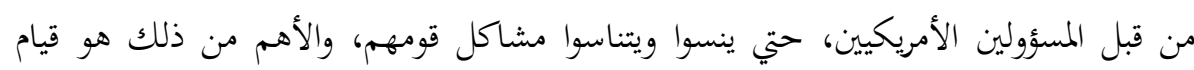

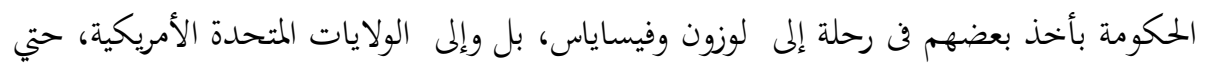

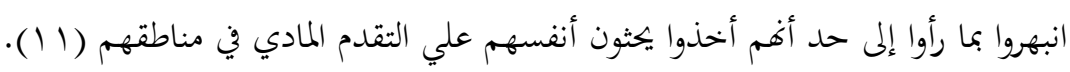
ولهذا كان هؤلاء(الزعماء المسلمون) سبباً فن نقل ثقافات الغربيين إلى بيوت المسلمين، وفتحوا بجالاً واسعاً للنصاري للعمل أو الاستيطان في مناطقهم، بل وكانوا سبباً فن وضع أبنائهم في مستنقعات الغزو الفكري والثقافي، مما أدي إلى الويلات والنكبات للشعب المسلم في الفلببن،

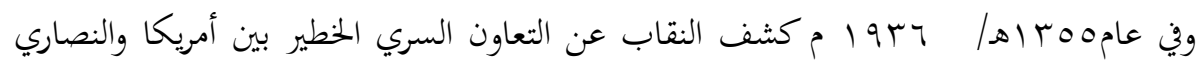
الفلبينيين من خلال تغيير الجهاز المختص بشؤون المسلمين في مينداناو وسولو من مكتب شؤون القبائل غير المسيحية، إلى مكتب شؤون القبائل الفلبينية ليوافق تغير وضع الحكم الأمريكي فن

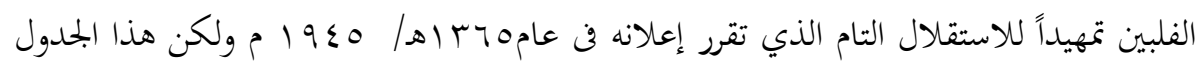


الزمني قد تأجل إلى يوليو 19 ا 1 م بسبب أنشغال الأطراف المعنية بالحرب العالمية الثانية(r I ).

وكذلك كانت إدارة فرانك كاربنتر في مينداناو وسولو مَدف إلى التكامل السياسي للمسلمين في الفلبين، فلقد سعى إلى سياسة جذب المسلمين في الفلبين من خلال توفير فرص سياسية واقتصادية واجتماعية مماثلة للفلبينين المسيحيين وعلي الرغم من حسن نية هذه السياسة في مينداناو وسولو فإنها لم تكن ناجحة كما كان يأمل الكثيرون من الناس في ذلك الوقت ويرجع ذلك إلى سببين رئيسين هما أولا: ازدراء الأمريكيين لغير المسيحيين في الفلبين في كثير من

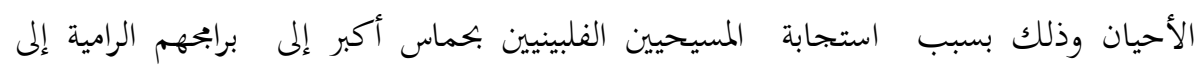
الديمقراطية، كما شجب الأمريكيون اثنين من الممارسات لدى مسلمى مورو وهما العبودية(إقامة الشعائر الدينية) وتعدد الزوجات وسعوا إلى تغييرها، ولكن المورو اعتبروها جزءاً لايتجزأ من

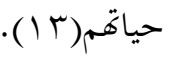

ولهذا شعر المورو(مسلمو الفلبين) بتلك الممارسات الغربية المسيحية التي تدعو للتنصير وبأها كانت توجد في غرائز المستعمرين الأمريكيين مثلهم مثل أسلافهم الأسبان، والتي استمرت في عهد الأسبان لقرابة ثلاثة قرون، ثانياً :قيام الأمريكيين بتولي بعض المسيحيين المناصب العليا، واتخاذ الأمريكيين مواقف لصالح المسيحيين الفلبينين منها تمليكهم العقارات وغيرها من بن الممتلكات غير المشروعة وخاصة في مينداناو ،وذلك علي حساب مورو(مسلمى الفلبين) بالإضافة إلى منحهم أراضٍ وإعفائهم من الإقرارات الضريبية وما إلى ذلك، وكان من نتيجة هذا الفساد عدم احترام صارخ لمورو و الإستيلاء علي ثروات أجدادهم، ولقد استمرت هذه

$$
\text { الممارسات من عديمي الضمير حتي اليوم (ع (). }
$$

كما لم يفعل الأمريكيين أى شئ يذكر لتحسين الأوضاع الاقتصادية للمسلمين رغم كثرة وعودهم، وكان التكالب علي هب الثروات الطبيعية فن الجنوب المسلم هدفًاً مشتركاً بين فئات ثلاث هى: الاحتلال الأمريكي، والرأسماليون الفلبيينيون، والساسة الفلبينيون المسيحيون ، وكان 
همْ الأمريكيون الأكبر هو فتح أرض عذراء لأنفسهم ولأصدقائهم ،وقد رأوا أن استقدام

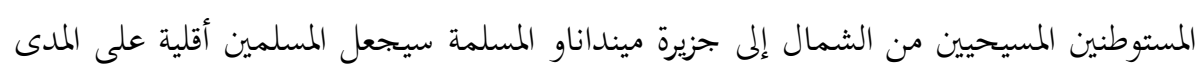
الطويل، بحيث يمكن عندئذ إهمالهم وبحاهلهم (10).

على أية حال مرت الفلبين إبان حكم الأمريكيين لها بعهدين : الأول عهد الحكم

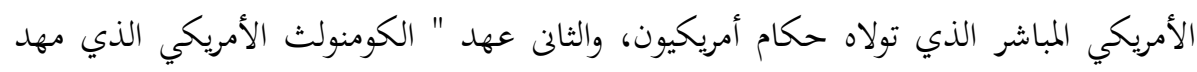

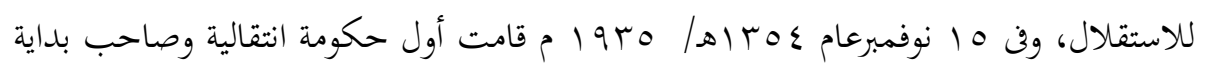
فترة الانتقال إجراء الاقتراع لاختيار أول رئيس للبلاد من العناصر الوطنية وكانت له كافة الهانة

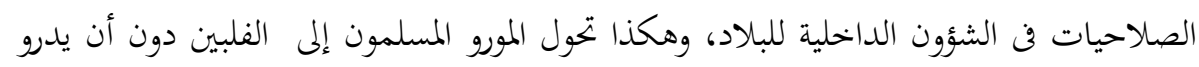

$$
\text { من أمرهم شيئاً (17) (1). }
$$

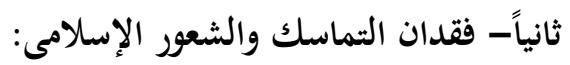

إن الإمبرياليين من الكاثوليك الغربيين، وخاصة ساسة الاحتلال الأمريكي الجدد، هم

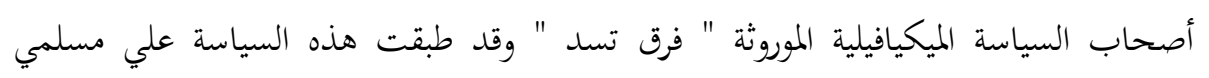
الفلبين بعلانية واضحة، وف خاية القرن الثالث عشر المجرى/ التاسع عشر الميلادي، كان مسلموا الفلبين بالرغم من انقسامهم فن عدة سلطنات وإمارات مستقلة، وبالرغم من الحدود الطبيعية التي كانت تفصل بينهم كانوا صفًاً واحداً خاصة عند الحرب ضد العدو المشترك، وكان الإسلام هو

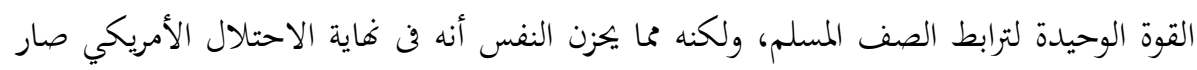
المسلمون فن فرقة وانقسام مروع يجهلون بعضهم البعض تماماً (V) ).

ولذلك كانت المأساة الكبري هى فقدان التماسك والشعور الإسلامي بين المسلمين، تلك

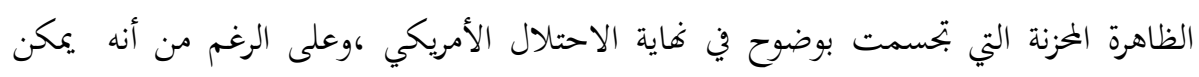

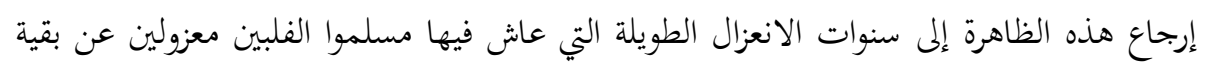

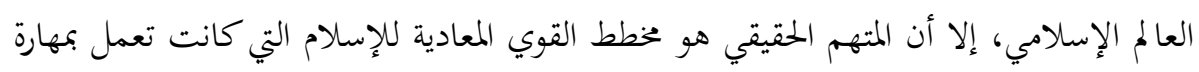
لتحقيق هذا الهدف فن النهاية، ومن بين القوي المعادية للإسلام بحانب الإمبريالية، والاستعمار 
الأسباني والاحتلال الأمريكي، والمئات من البعثات التنصيرية الكاثوليكية والبروتستانتية وكافة المذاهب فضلاً عن السياسة التي اتبعت في البحال السياسي، والاجتماعي، والثقافي، وهي الصهوينة العالمية التي تعد من هذه القوي المعادية جميعاً تأتي لتتزعم الموجة المعادية الجحديدة، وأيضاً تأتي كالأفعي اللدود ضد الإسلام(1) (1).

ثالثاً - فقدان السيادة الإسلامية على معظم الإمارات والسلطنات:

قبل وصول الاحتلال الأمريكي فن غاية القرن الثالث عشر الهجرى/ التاسع عشر الميالادي، كانت هناك عدة سلطنات وإمارات إسلامية في جنوب الفلبين حيث ظلت هذه الإمارات مستقلة دون أن تزمها أسبانيا، وعلي وجه الخصوص فى جزيرة مينداناو، وبجموعة جزر سولو، وكانت أكبر سلطنة في ذلك الوقت هي سلطنة سولو التي جمعت جزيرة باسيلان وجزءاً من شمال بورينو الذي يُعرف حالياً باسم ولاية صباح(9 1) فن الاتحاد الماليزي، ويلي هذه الأهمية سلطنة مينداناو والتي كانت تحكم الوادي الجنوبي من مقاطعة كوتاباتو والجزء الجنوبي من شبه جزيرة زامبونحا، يلي هذه سلطنة بويان التي كانت أراضيها الواسعة تضم معظم الأجزاء الجنوبية في مينداناو وسلطنة كابوتنالان التي كانت تحكم السهول الوسطي في كوتاباتو وسلطنتي لانو وكاجيان المتحدتان فيدرالياً وإمارات أخرى كانت كلها باستثناء سولو ماتبقي من السلطنة الأولي للبطل المسلم العظيم السلطان ديبرتوان قدرات قاهر المستعمرين الأسبان والكاثوليك في الفلبين ،وكانت كل هذه مستقلة ذات سيادة ،ولكن دوامة الحكم الأمريكي كما ذكرنا من قبل بلا شعور أو إدراك ابتلعتها جميعاً (· (广).

حيث أنشأت الولايات المتحدة الأمريكية في عام ع ا9 ام وزارة خاصة بشؤون تلك المناطق وهى سولو، ولاناو، ومينداناو، وزامبونجا ،وأبى ،وغوش ،وبوكيدتون ،وعزلت تلك المناطق

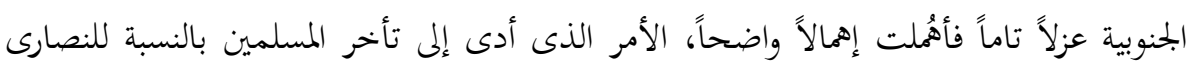
الذين يتلقون دعم الاستعمار المسيحى (Y) 


\section{رابعاً - الاستيلاء على الأراضى الزراعية:}

في بداية الاحتلال الأمريكي كانت بحموعة جزر مينداناو وسولو بأكملها تخضع من الناحية النظرية لملكية المجتمعات الإسلامية بناء علي مبدأ السيادة التي تتمتع بها، غير أنه بعد فقدان السيادة وما تبعها من فقدان الحكم، فضلاً عن فرض القانون الأمريكى في نظام حيازات

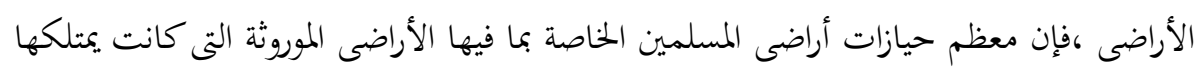
أجدادهم والموارد الاقتصادية الأخرى ،ضاعت وسقطت في أيدى الكاثوليك الأكثر ثراء ،وعلماً

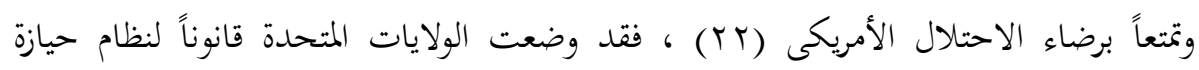
الأراضي، كان من نتيجته فقدان المسلمين لكثير من الأراضي التي ورثوها عن أجدادهم وسقطت في أيدي المسيحيين الكاثوليك (rT).

حيث ينص هذا القانون على أن جميع رخص امتلاك الأراضي غير المصدق عليها من أسبانيا تعد ملكية عامة، وهذا يعني أن أراضي المسلمين تُصبح ملكاً للحكومة الأمريكية ؛لذلك رلك زادت الهجرة الصليبية إلى الجنوب بسبب هذا القانون وخاصة بعد صدور قانون آخر يُيبح لكل لكائل مهاجر امتلاكك ؟ هكتارات من الأرض، بينما المواطن الأصلي في الجنوب يجق له امتلاك ع هكتارات، وجاء النصاري بأسماء شركات وهمية لامتلاك أراضي المسلمين (ع ب).

وإحقاقاً للحق فإننا لوقارنا بين معاملة الأسبان ومعاملة الأمريكيين للمسلمين فإننا سوف بند فرقاً،حيث إن الأمريكيين قد تساموا فن آخر الأمر مع المسلمين والشعب الأمريكي يستمع إلى كل ذى رأي، وعلى أستعداد لقبول كل رأي يؤيده العقل والمنطق، ويذكر مدير المركز الثقاف الإسلامي بواشنطون أن مواقف كثيرة كانت له مع الأمريكيين، إن دلت علي شئ فإنها تدل دلالة

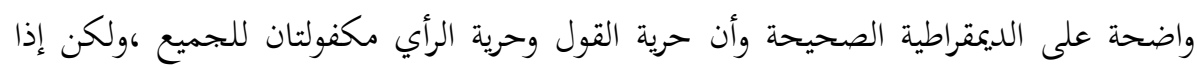
أردنا أن نقوم من الناحية الموضوعية بالإصلاحات التي قدمها الأمريكيون للمسلمين خلال فترة

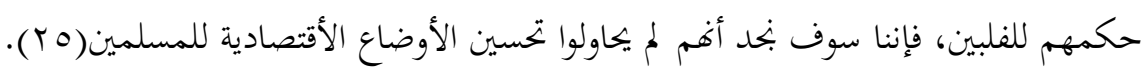


وربما كان ذلك بسبب عدم ثقة المسلمين بالأمريكيين الذين كانوا ينظرون إليهم كخلفاء للأسبان في الحكم الاستعماري ،ومن المتمل أن يكون سبب فشل الأمريكيين فن القيام بإصلاحات اقتصادية جذرية في أراضي المسلمين هو نفس السبب الذى أدى لفشلهم في الأجزاء الأخرى من الفلبين ،ومع ذلك اهتم الأمريكيون مع بعض أصدقائهم من الساسة ، والرأسماليين الفلبينيين فن استصلاح أراضٍ جديدة لهم ولأصدقائهم فف جنوب الفلبين ،وكانت هذه سياسة قُصد بها إسكان عدد كبير من المسيحيين فن مينداناو حتى يصبح المسلمون أقلية لاقيمة لها ،وآثار هذه السياسة ظاهرة للعيان حتى الآن(† ؟). كما استفادت الولايات المتحدة الأمريكية من أراضى المسلمين وذلك بإنتاج كميات كبيرة من السكر الفلبيني وإدخاله للولايات المتحدة وبدون جمارك وبدأ في العشرينات من هذا القرن ضغط من قبل أعضاء الكونجرس الأمريكي الذين مثلوا ولايات غربية في أمريكا، مثل ولاية

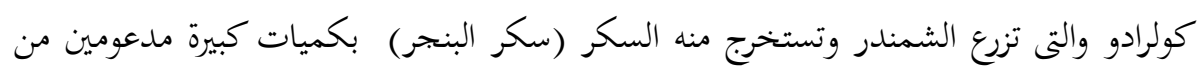

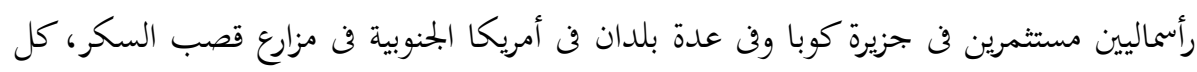
هؤلاء طالبوا في الكونخرس الأمريكي فرض الجمارك علي السكر المستورد من الفلبين، وأخيراً وما

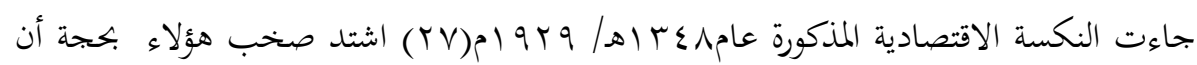
فرض الجمارك علي سكر الفلبين سيفيد في تقليل البطالة فن الولايات الغربية من أمريكا ويحسن

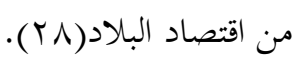

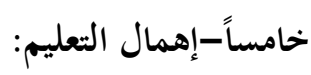

لقد شعر بعض المورو(مسلمو الفلبين) بالمرارة والقسوة من القوانين والعقوبات التى فرضتها الولايات المتحدة الأمريكية ضد العبودية(إقامة الشعائر الدينية) وكذلك فرضهم الضرائب علي مورو، ولكن الأهم من ذلك كله هو نظام التعليم الذى كانت ترعاه الولايات المتحدة الأمريكية، فلقد كانت وجهة نظر المورو أن هذه المحاولة هي الأكثر ضراوة حيث إن الأمريكان قد اهتموا بالمدارس من أجل غرس التعاليم المسيحية أوالقيم المسيحية التي سوف تقوض وتمدم عقيدقم 
الإسلامية، لقد كان ذلك شكلاً من أشكال الإبادة الجماعية النفسية، ومحاولة من أجل القضاء علي الهوية الدينية والثقافية للمورو؛ ونظراً لأفم كانوا وبوضوح أقلية فليس لهم الحق في المطالبة بالتعليم ،فلقد تم منع أطفال مورو من الذهاب إلى المدارس وبقاء أبنائهم في المنزل (9 ب).

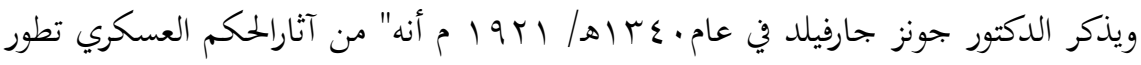
التعليم تدريجيا في أراضي المسلمين؛ لأن الوضع كان يحتاج إلى تأسيس النظام للمدارس العامة مقارنة لما في مقاطعات النصاري التي ابتدأ التعليم فيها منذ عقدين من الزمان ،غير أن الحكومة

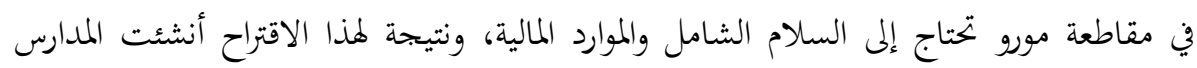
العامة في عامع بس اهـ/ 1910 م في منطقة مينداناو وسولو تحت إشراف المكتب التعليمي، الذي بنح في تطوير المدارس العامة الضخمة علي غرار نظام المدارس النصرانية، ومن الملاحظ

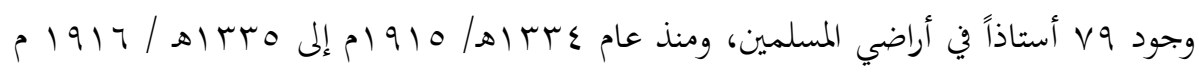

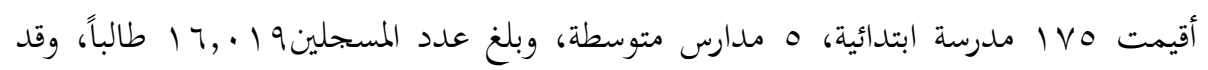

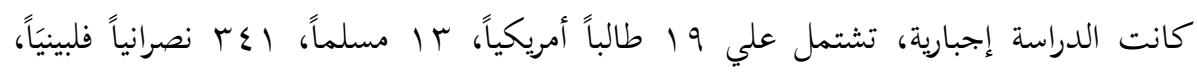

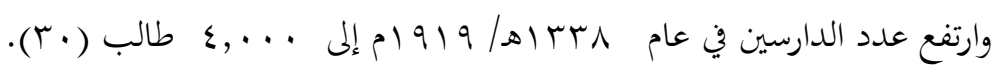
ويذكر أيضاً الدكتورجونز جارفيلد أيضاً أنه" مادامت المدارس الموروية أنشئت تحت إشراف المكتب التعليمي فإن مئات المدرسين من النصاري سيرسلون إلى المناطق الجنوبية لافتتاح المدارس الابتدائية هناك، وفي أقل من العقدين الماضيين أدهشت الولايات المتحدة الأمريكية العالم بإرساها . ـ مدرس أمريكي إلى الفلبين لتدريس الحضارة الحديثة، وقد أعطت هذه المدارس صدي للمسلمين حيث أرسل سلطان سولو جمال الدين القرم الثاني بنته إلى الولايات المتحدة الأمريكية بعد أن أتمت تعليمها في المدارس العامة في زامبونحا (آب). ويذكر الدكتور فرانك لوباش: من العجيب في مينداناو وسولو وهولأول مرة في التاريخ للدين المتعصب و" المشار إليه الدين الإسلامي" ذهاب المسلمين إلى المدارس تحت إشراف المدرسين النصاري، والأغرب من ذلك أن هؤلاء أرسلوا بناتم إلى المدارس النصرانية مع أن إن 
مشايخهم يرفضون ذلك ؛لأنه مخالف لدينهم، ومن العجيب أيضا أنه حينما رأينا السلطان وهو من المتدنين بالإسلام كما هو قائد للمسلمين أمر بنته أن تعلم \&؟ بنتا في المدرسة وأن مئات النساء لهن طموحات في التعليم مثل الرجال، الكل يعرف أن المورو حدث لهم نوع من التقدم العجيب، وهم مفخرة وموضع إعجاب لأمريكا، إذا لم يسبق في تاريخهم (المسلمين) أن بند أحداً منهم مستعد للدخول فن الديانة النصرانية (r (r). ونظراً لوجود التعليم تحت إشراف الإرساليات التنصيرية المسيحية فهذا جعل الثقافة الإسلامية تتقهقروالجهل يسود بين أبناء المسلمين(r/).

ونتيجة لتلك السياسة المتعصبة وتعرض المسلمين للقهر والاعتداء والتنصير،أسس المسلمون الجمعيات الإسلامية التى تعمل على تدريس العلوم الإسلامية واللغة العربية وإصدار

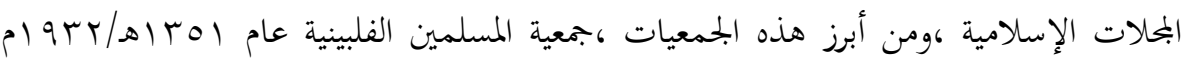

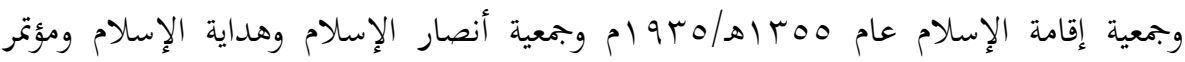
الإسلام والنهضة الإسلامية والتربية الإسلامية والمؤتمر الإسلامى وجمعية مسلمى سولو (ع؟ب). 


\section{الخاتمة}

الحمد لله الذى وفقنى فن هذ البحث ويسر إتمامه، وفى هاية هذا البحث أذكر أهم النتائج التى توصلت إليها من خلاله، وهى كما يلى:

1- أن الاحتلال الأمريكي ترك آثاراً سلبية على الفلبين بشكل عام وعلى المسلمين بشكل خاص فن جنوب الفلبين ،على الرغم من قصر الفترة الزمنية مقارنة بالاستعمار الأسبانى. إلا أن الولايات المتحدة الأمريكية كانت أكثر فعالية على جزر الفلبين.

ץ-لقد استخدم الاحتلال الأمريكي أسلوب الحنديعة والمكر والخبث السياسى وعن طريق ذلك استطاع الأمريكان كسب عواطف الزعماء المسلمين فن الجنوب ودخلوا المناطق الإسلامية الجنوبية ولم ييق لزعماء المسلمين أية سلطة في المناطق الجنوبية سوى السلطة الروحية واستفادوا من بحربة الأسبان القمعية ضد الجزر الجنوبية ثم لجأ الأمريكان إلى سلاح آخر وهو السلاح الثقافى لكسب لئب عاطفة وشعور المسلمين و ذلك بسبب أن سلاح القوة التي لجأ إلية الأسبان؛ وكذلك الأمريكان في المراحل الأولي لم تفدهم ولم يجلب لمم بحاحاً، أما السلاح الثقافي فقد كان أشد تأثيراًحيث يحارب العقل ويغير الأفكار والاتحاهات لذلك انصب اهتمام الأمريكان بالتعليم.

r-حاول الأمريكيون تنصير المسلمين بالإكراة والقوة ؛ونتيجة لذلك تكونت جمعيات إسلامية

$$
\text { للوقوف فن وجه الاحتلال الأمريكي . }
$$

ع - مرت الفلبين في أثناء احتلال الولايات المتحدة الأمريكية لجزر الفلبين بعهدين ،العهد الأول هو الحكم المباشر للولايات المتحدة الأمريكية لجزر الفلبين، والثانى عهد الكومنولث الذى مهد

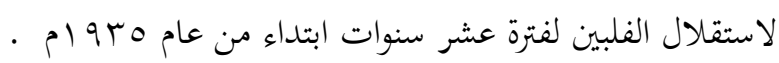

ه-أن المقاومة المستمرة لمسلمى الفلبين في فترة الاحتلال الأمريكي أدت إلى تناقص عدد المسلمين و تعطيل تقدمهم في جميع مناحى الحياة المختلفة. 
צ-من الآثار الضارة أيضاً للاحتلال الأمريكى للفلبين هو فقدان السيادة والشعور والتماسك الإسلامى ،وكذلك قيام الاحتلال الأمريكي بالسماح لليهود للدخول إلى جزر الفلبين. V- أن الدمار والخراب الذى خحلفته الحرب الطويلة بين المسلمين والأمريكان أعاق أعمال الدعوة ونشر الإسلام، كما حرم شعب مورو من أية فرصة لمتابعة العمل على إقامة بحتمعهم على أسس برس روحية اجتماعية تتفق مع أوامر القرآن.

1- أن تعاطف مسيحيى الشمال مع الاستعمار بشكل عام والاستعمار الأمريكي بشكل خاص وتدهور الأوضاع السياسية والاقتصادية والاجتماعية للمسلمين، كان من أكبر الأسباب لوجود الصراع بين الجانبين.

9-قامت اليابان بعمل هجوم على ميناء بيرل هاربور التابع للولايات المتحدة الأمريكية وذلك لوقوف الولايات المتحدة الأمريكية عقبة في طريق التوسع الاستعمارى اليابانى فن جنوب شرق آسيا واحتلال جزر الفلبين عام بـ 9 ام،ولكن الولايات المتحدة الأمريكية استطاعت العودة مرة أخرى وانتصرت على اليابان فن الحرب العالمية الثانية وقررت إعطاء الفلبين الاستقلال عام

وأما عن التوصيات التى أوصى بها ،وهى لابد من الدخول في هذا المحال وهذا النوع من الموضوعات التى أخذت شيئاً من الإهمال، ولذلك لابد من التهئ لتوكيز عليها. 


\section{هوامش البحث:}

(1) محمد خليفة بالقاسم البرهمي :أثر الأقليات فن استقرار الدولة القومية (دراسة حالة )الأقلية

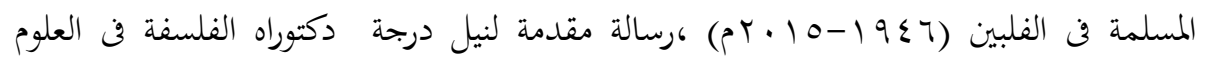

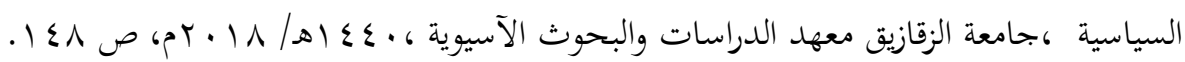
(r) السكرتير التنفيذي لجمهورية الفلبين وأحد كبار وزراء الحكومة الحالية في الفلبين، أحمد ألونتو

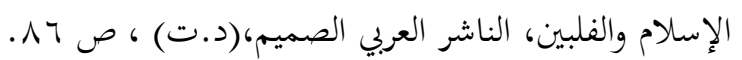

$$
\text { (广) أحمد ألونتو: المرجع السابق، ص NV،人T. }
$$

(§) محمد حسن راشد الزهراني: مشكلة الأقلية المسلمة في جنوب جزر الفلبين، رسالة ماجستير،

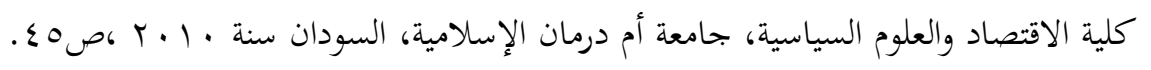

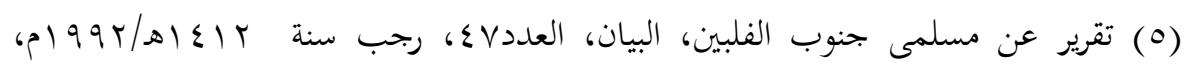
ص מ מ (7) أحمد ألونتو: المرجع السابق ،ص 11.

(V) وليام هاورد تافت:ولد عام Vم ا ام بولاية اوهايو، عضو الكنيسة التوحيدية ،وانتخب عن الحزب الجمهوري لفترة رئاسية واحدة عام 9 ـ9 ام، وتوف بواشنطن عام ·ـو ام، وهو الرئيس السابع والعشرين للولايات المتحدة الامريكية، أصبح أول حاكم مدنى للفلبين ثم أصبح وزير للحرب في عهد الرئيس ثيودور روزفلت، رافت غنيمى الشيخ: امريكا والعالم فن التاريخ الحديث

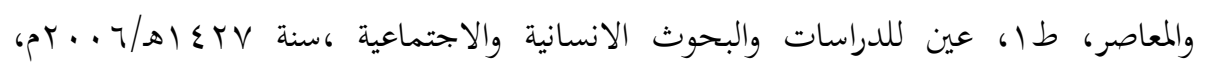

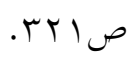

(^) جميل عبدلله المصرى: حاضر العالم الإسلامى ،ط • (1)ج|،كتبة العبيكان ،الرياض سنة

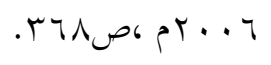


(9) خالد الأصور: النزعات الأنفصالية فن شرق أسياً ،بحلة الديمقراطية (وكالة الأهرام )بحلد؟،

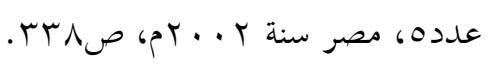

.T.J.S George: Revolt in Mindanao (Kuala ( $1 \cdot$ ) lumpur :oxford university press, 1980,pp59-61.

:The history of an island

Delor Angeles : mindanao

pedro Press1964,pp67-68

Davao city :Philippines ,san

: opcit ,p68.

Delor Angeles : mindanao

Gowing, peter G.:Muslim Filipinos - Heritage and (Ir) Horizon ,Quezon city ,New day publishers, 1979,,p339. Gowing ,peter

G :opcit,p339

(10) محمد يوسف عدس: الإسلام والمسلمون في الفلبين، ج، بطبعة

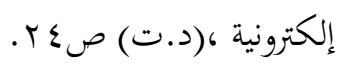

(7 (17) مصطفى مؤمن: عذراء ماليزيا (الفلبين)، ط ا، دار التراث العربي، ليبيا سنة

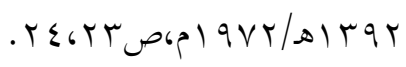

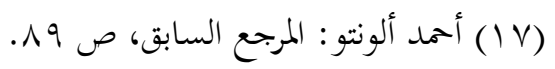




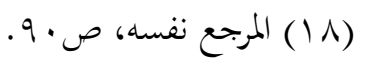

(9 (19) صباح: هى توجد في الجزء الشمالى من جزيرة بورينو، وتبلغ مساحتها حوالى

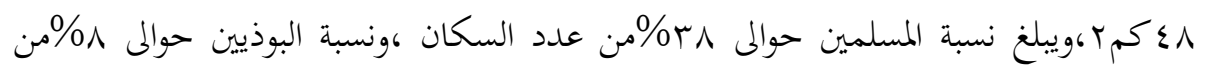

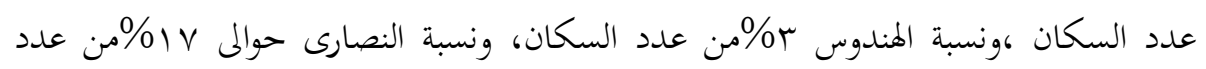
السكان ،وعبدة الأرواح حوالى هب\%\%من عدد السكان، وقد انضمت إلى الاتحاد الماليزى فور إنشائه وعرفت بولاية صباح ويتكون سطحها من سهول وتلال عادة، وتمتد السهول حتى الشريط

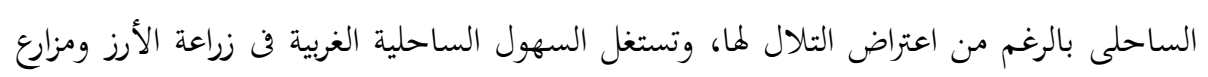

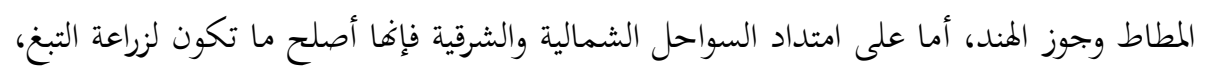
محمد عبد الغنى سعودى: آسيا فن شخصية القارة وشخصية الأقاليم، مكتبة الأنجلو المصرية،

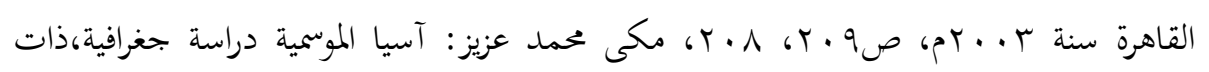

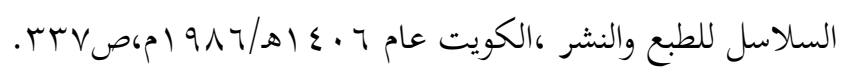

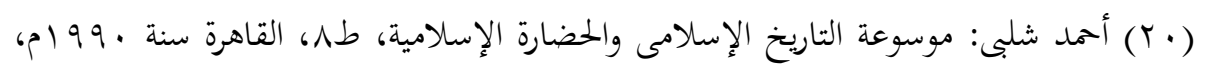

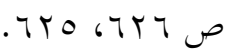

(Y)(Yممود أحمد قمر :تاريخ وحضارة الإسلام فن آسيا والشرق الأقصى ،ط ا،كلية الأداب،

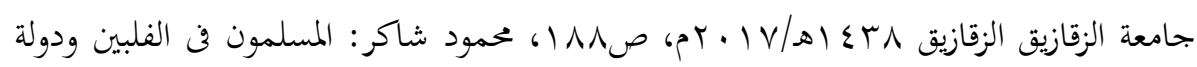

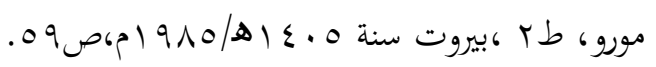
( أحمد ألونتو:المرجع السابق ،ص 19 (Y)

(YT)

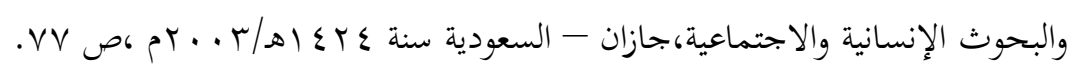

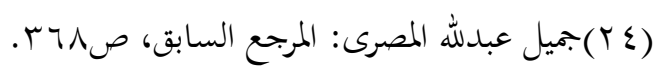


(Y0) محمد عبد القادر أحمد: المسلمون في الفلبين ،مطابع الناشر العربى ،القاهرة ،سنة .

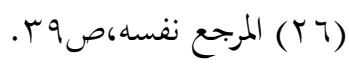

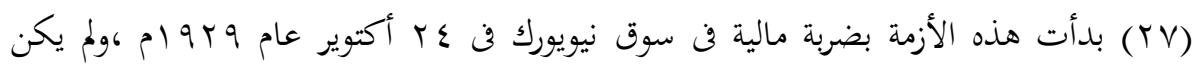

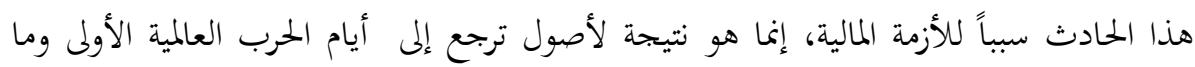
بعدها، وعندما تولى روزفلت أمور بلاده عمل علي أن ينقذ بلاده من هذه الأزمة وذلك عن

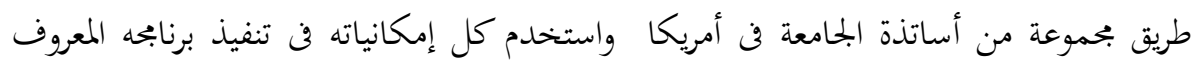
باسم(العهد الجديد)، رأفت غنيمى الشيخ: أمريكا والعالم في التاريخ الحديث والمعاصر، صع ال 1)، .114

(YN)

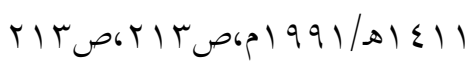

-Ralph B Thomas :muslim

But Filipino the integration of Philippine muslim ,19171946 ,university of . Pennsylvania,1971,p60. our mandate over land, the muslim world, $(r \cdot)$

Jones Garfield::Moro volx1 1921, New York:1966 ,p34.

: opcit ,p35

Jones Garfield 
Farnk Laubach : Islam in the Philippines, the muslim (rr) world volx11 1922,new york 1966,pp64-65 (Tr محمود قمر: تاريخ وحضارة الإسلام فن آسيا والشرق الأقصى،ص1/11، امحمود شاكر: المسلمون في الفلبين ودولة مورو، صوه ه. (§؟) هيا بنت عبدالمسسن محمد البابطين: أحوال الأقليات المسلمة في الفلبين في النصف الثاني من القرن العشرين، بحلة العلوم الأنسانية والأجتماعية، العدد.ء؛، السعودية أبريل سنة $.49106 r+17$

قائمة المراجع العلمية

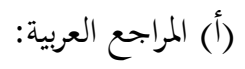
1-أحمد شلى: موسوعة التاريخ الإسلامى والحضارة الإسلامية ،طم، القاهرة سنة .99 19م ب-أحمد ألونتو: الإسلام والفلبين ،الناشر العربي الصميم ،د.ت) r-تقرير عن مسلمى جنوب الفلبين، البيان ، العددلع، رجب سنة rإـاهـ/99 ام ع - جميل عبدلله محمد المصرى :حاضر العالم الإسلامى ،ط • (،ج|،مكتبة العبيكان ،الرياض

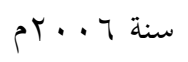
ه-خالد الاصور: النزعات الانفصالية في شرق آسيا ،بحلة الديمقراطية (وكالة

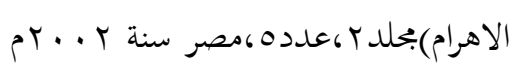

צ-رافت غنيمى الشيخ: أمريكا والعالم في التاريخ الحديث والمعاصر، الطبعة الأولى، عين

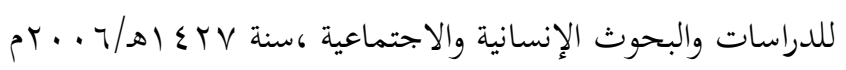


V-عبد الشافي غنيم عبد القادر وأخر :قضايا إسلامية معاصرة ،عالم الكتب ، الكويت عام.191 1م

^-فايز صالح أبوجابر: الاستعمار في جنوب شرقى اسيا ،طا،دار البشير ،عمان ،سنة 1991/81 1911

9- محمود قمر: الإسلام والمسلمون في شرق وجنوب شرق آسيا، طا، عين للدراسات

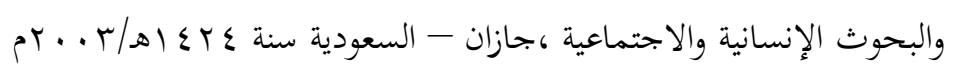

• 1-محمود أحمد قمر: تاريخ وحضارة الإسلام فن آسيا والشرق الأقصى، طا،كلية الأداب

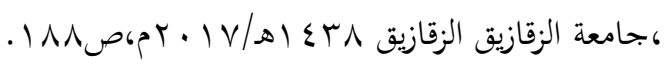

|ا-محمود شاكر: المسلمون في الفلبين ودولة مورو، طب، بيروت سنة $.090601910 / 81 \leq .0$

با بمحمد عبد القادر أحمد: المسلمون فن الفلبين ،مطابع الناشر العربى ،القاهرة ،سنة •ـ19 ام با ا-محمد يوسف عدس: الإسلام والمسلمون في الفلبين، ج، كطبعة إلكترونية، (د.م)،بدون تاريخ.

ع ا- محمد خليفة بالقاسم البرهم: أثر الأقليات في استقرار الدولة القومية (دراسة حالة )الاقلية

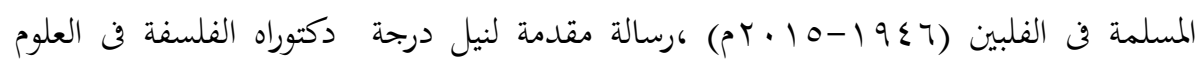

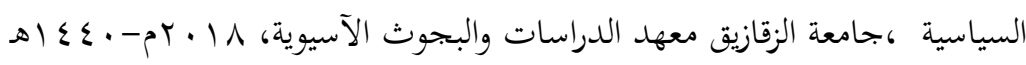
1 - بحلة الوعى الإسلامية: المسلمون فن الفلبين، الكويت، سنة • و ام 17

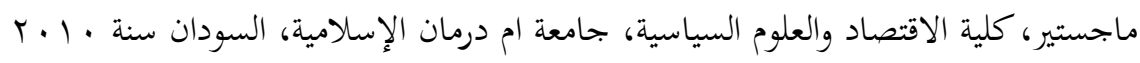


I V

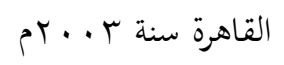

-11 - مكىى محمد عزيز : آسيا الموسمية دراسة جغرافية،ذات السلاسل للطبع والنشر ،الكويت

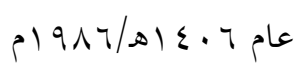

9 1-مصطفى مؤمن: عذراء ماليزيا (الفلبين) ،طا، دار التراث العربي، ليبيا سنة $.619 V T / D 1 r q r$

• ץ-هيا بنت عبدالخمسن محمد البابطين: أحوال الأقليات المسلمة في الفلبين في النصف الثان من

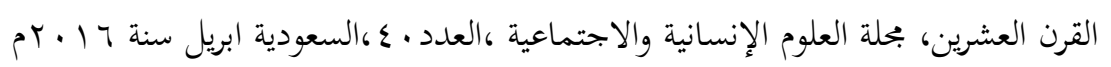

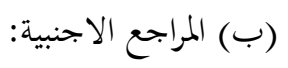

1 - : mindanao :The history of an island

Delor Angeles

Davao pedro Press1964

city :Philippines ,san

2- Farnk Laubach : Islam in the Philippines, ,the muslim world volx11 1922,new york 1966 3-Gowing, peter G.:Muslim Filipinos - Heritage and Horizon ,Quezon city ,New day publishers,1979

:: our mandate over Moro land,the muslim worl, 4-Jones Garfield 
New York:1966

$$
\text { volx1 }
$$

5-Ralph B Thomas :muslim But Filipino the integration of Philippine muslim ,1917-1946, university of Pennsylvania, 1971

6-T.J.S George: Revolt in Mindanao (Kuala lumpur :oxford university pres)s, 1980 